\title{
Reply to Letters to the Editor re: Low-dose computed tomography of the paranasal sinus and facial skull using a high-pitch dual-source system-First clinical results
}

\author{
Boris Schell
}

Received: 3 January 2011 / Accepted: 31 January 2011 / Published online: 12 March 2011

(C) European Society of Radiology 2011

\section{Dear Editor,}

We appreciate the thorough analysis made by the authors of these two letters with regards to our original publication. In our study we noted amongst other things decreased CTDI ${ }_{\mathrm{vol}}$ (volume CT weighted dose index) and DLP (dose length product) values when using a second $\mathrm{X}$-ray with increased table feed (dual source high-pitch mode).

First, we agree that calibrations of CTDI ${ }_{\mathrm{vol}}$ values of the device are done on various phantoms by the vendor (head with $16 \mathrm{~cm}$ respectively body/chest with $32 \mathrm{~cm}$ in diameter). From that background, the concerns the authors raise appear to be coherent. Indeed our very own measurements given by the CT protocol show a significant lower dosage when using the high pitch-mode. Since that calibration was done on a body phantom and the mode was used in a head region, a correction of DLP values seems to be necessary. The electrocardiogram-gated highpitch examinations of the heart quoted by the authors were done using automated tube current control which raises the tube current with increasing pitch. This is contrary to our examination protocol where tube current output remains stable since no automatic exposure control was used. Yet to display the actual radiation dose of the proposed examination techniques, specific measurements of a head phantom equipped with thermoluminescent dosemeters (TLDs) would be necessary. This would also be necessary to define the contribution of the bowtie filter mentioned by the authors. However, this data is not available yet. We suggest that further studies should be performed analyzing the influence on the different phantoms and experiments using different shaped filters.

The letters also draw attention to another issue regarding dose values given by $\mathrm{CT}$ devices. $\mathrm{CTDI}_{\mathrm{vol}}$ and DLP are routinely used to estimate radiation exposure of the patient. According to the "European Guidelines on Quality Criteria for CT" stated by the European Commission in 1999 dose reference levels are also given in terms of $\mathrm{CTDI}_{\mathrm{vol}}$. However this value indicates energy imparted to a volume irrespective to the examined spatial pattern. This may not only lead to improper dosage results given by CT devices (e.g. with varying patient diameter) but can somewhat show falsely low results when using torso examination protocols for peripheral regions. Subsequently it is questionable whether the actual dose values shown by $\mathrm{CT}$ devices might nowadays function as a reasonable and reliable tool to estimate effective dose or to monitor examination protocols in terms of dose reference levels.

\footnotetext{
B. Schell $(\square)$

Department of Diagnostic and Interventional Radiology,

Clinic of the Goethe University,

Haus 23 C UG, Theodor-Stern-Kai 7,

60590 Frankfurt, Germany

e-mail: boris.schell@googlemail.com
} 